\title{
Continuous Refractive Index Sensing based on Carbon-Nanotube-Deposited Photonic Crystal Fibers
}

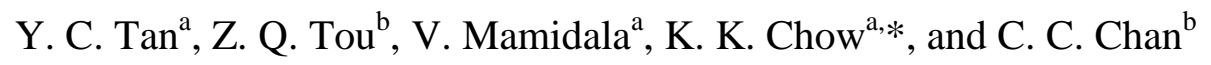 \\ ${ }^{a}$ School of Electrical and Electronic Engineering, \\ Nanyang Technological University, 50 Nanyang Avenue, Singapore 639798 \\ ${ }^{\mathrm{b}}$ School of Chemical and Biomedical Engineering, \\ Nanyang Technological University, 70 Nanyang Drive, Singapore 637457 \\ *E-mail: kkchow@ntu.edu.sg
}

\begin{abstract}
We present a carbon nanotubes (CNTs) deposited photonic crystal fiber (PCF) featuring a MachZehnder interferometer configuration for refractive index (RI) sensing applications. The high RI of the CNTs deposited on the surface of the PCF not only enhances the interaction of the evanescent waves of the cladding modes with the ambient environment around the fiber, but also modifies the sensing scheme to that of intensity variations. Such a modification makes the sensor susceptible to power fluctuations from the optical source but allows the sensor to gain immunity to free spectral range limitations which is commonly found in PCF-based sensors. As such continuous and repeatable measurements can be obtained for the range of RIs being measured. The sensor registered a sensitivity of $19.4 \mathrm{~dB} / \mathrm{RIU}$ within the RI range of 1.33 to 1.38 and a sensitivity of $24.2 \mathrm{~dB} / \mathrm{RIU}$ within the RI range of 1.38 to 1.42 . Since there is no mechanical modification of the overall structure of the sensing element, the fiber retains its mechanical
\end{abstract}


strength which makes it viable for practical applications. The experimental results are found to be consistent with the modeling of the sensor's behavior.

Keywords: optical fiber sensor; carbon nanotube; refractive index sensor; photonic crystal fiber

\section{$\underline{\text { Introduction }}$}

Refractive index (RI) sensing is an important process in many biological and chemical environment applications. Due to their light weight, immunity to electromagnetic interference, cost efficiency, flexible design, and multiplexing capabilities, optical fibers have generated a lot of research interest as RI sensors. Most of the proposed sensors involve modifying a short section of the optical fiber to obtain a highly sensitive sensing element. Some of these modifications include tapering [1-3], multimode interference [4-6], fiber core offset [7, 8], grating inscription $[9,10]$, and using specially fabricated optical fibers like photonic crystal fibers (PCFs) $[11,12]$. These sensors usually register variations in the ambient environment through changes in wavelength or intensity in their output spectrum. However, some of these modifications result in the weakening of the overall structure of the fiber, which would lead to a lack of robustness of the sensing element. Also, the RI sensing schemes using LPFG or PCF usually involve variations in wavelength of the output spectrum in response to variations in the ambient environment surrounding the sensing element. These sensors are usually very sensitive to perturbations in the ambient environment and are immune to any fluctuations in source intensity as long as there is a sufficiently high extinction ratio in the spectrum for detection. However, due to the nature of the output spectrum, such a scheme is usually susceptible to free spectral range (FSR) limitations, and continuous real time measurements cannot be obtained over a wide range of RIs. In recent years, there has been an increase of research interest in the 
fabrication of fiber sensors with deposition of a thin film over the sensing element to enhance its sensing capabilities. This usually involves depositing a thin layer of material over the sensing element such as a long period fiber grating (LPFG) [13-16], on a fiber end-face [17-24] or over a PCF [25-28]. A suitable material is first selected based on its optical properties corresponding to the particular ambient environment, and is deposited through various physical or chemical methods onto the fiber. The optical properties of the material will vary according to the ambient environment and cause a variation in the output of the sensing probe. However, the deposition techniques used usually involve highly precise equipment or a long fabrication duration due to the extensive amounts of chemical processes involved. Recently, our group has reported thefabrication of a LPFG deposited with a CNT thin film using spray coating method for RI sensing [29].

In this paper, a CNT-deposited PCF featuring a Mach-Zehnder interferometer (MZI) configuration for RI sensing is proposed. Conventional PCFs featuring a MZI configuration are fabricated by splicing a short length of PCF (the sensing probe) between two single-mode fibers (SMFs) [11]. The choice of a PCF is to fulfill the robustness requirement since such a scheme does not involve structural modifications which would weaken the fiber. Compared with other fiber devices like LPFGs, the adopted PCF is relatively temperature insensitive. Hence, it would not be affected by variations in temperature in the ambient environment, making it more versatile for RI sensing applications with fluctuating temperature conditions. The choice of CNTs as thin film material was due to its suitability for deposition by the spray coating technique as well as its compatibility to silica fibers [30]. Due to their tiny one-dimensional structure, CNTs can be easily dispersed through a nozzle and can adhere and bond strongly to any silica surface upon deposition. Previous reports have shown that CNTs could be suitable materials for the detection 
of hydrogen at cryogenic temperatures [17] and volatile organic compounds [18-20], indicating their versatility as a thin film material. For the case of the PCF, the CNTs act as a film of high RI and were deposited onto the PCF to form the sensing element using the spray coating method [30]. Hence, variations in the RI of the ambient environment resulted in a change in intensity of the spectral dips rather than a shift in wavelength which allowed the sensing element to overcome the FSR limitations. Such a modification would allow the sensor to gain immunity to FSR limitations and be subjected only to intensity fluctuations from the optical source which can be easily overcome by introducing additional power compensation techniques. The fabricated CNT-deposited PCF exhibited a sensitivity of $19.4 \mathrm{~dB} / \mathrm{RIU}$ within the RI range of 1.33 to 1.38 and a sensitivity of $24.2 \mathrm{~dB} / \mathrm{RIU}$ within the RI range of 1.38 to 1.42 .

\section{Sensing Principle}

A schematic diagram of the proposed sensing element is shown in Fig. 1(a). A short length of PCF is sandwiched between two SMFs, forming a MZI configuration. Two collapsed regions lie at the SMF-PCF and PCF-SMF transition regions. With this structure, the fundamental mode entering the collapsed regions from the input SMF would be diffracted $[11,31,32]$. This is due to the PCF no longer being a SMF as there is no core-cladding structure at that region. As shown in Fig. 1(a), the fundamental mode which is indicated by the solid arrow broadens and excites some of the cladding modes, which are represented by the dashed arrows. Since the propagation constants of the fundamental mode and the excited cladding modes in the PCF are different, there would be a phase difference as the modes propagate along the PCF. The core and cladding modes will propagate along the PCF before interfering and recombining at the second collapsed 
region and further propagating in the output SMF. The total intensity at the output SMF can be expressed as [33]:

$T=I_{\text {core }}+\sum_{i=1}^{n} I_{i}+2 \sum_{i=1}^{n}\left[\left(I_{\text {core }} I_{i}\right)^{1 / 2} \cos \frac{2 \pi\left(n_{\text {core }}-n_{i}\right) L}{\lambda}\right]+2 \sum_{i=1}^{n-1} \sum_{j=i+1}^{n}\left[\left(\mathrm{I}_{i} \mathrm{I}_{j}\right)^{1 / 2} \cos \frac{2 \pi\left(n_{i}-n_{j}\right) L}{\lambda}\right]$

where $I_{\text {core }}$ is the intensity of the core mode, $n$ is the number of excited cladding modes, $I_{i}$ and $I_{j}$ are the intensities of the $i^{\text {th }}$ and $j^{\text {th }}$ order cladding modes, respectively. $n_{\text {core }}, n_{i}$ and $n_{j}$ are the effective RIs of the core mode, $i^{\text {th }}$ order cladding modes and $j^{\text {th }}$ order cladding modes, respectively. $L$ is the length of the PCF fiber and $\lambda$ is the operating wavelength of the optical source.

As indicated by the dotted arrows in Fig. 1(a), the sensing mechanism relies on the evanescent waves of the cladding modes reaching the external surface of the PCF and interacting with the ambient environment [11]. This results in changes to the effective RI of the cladding modes as the RI of the ambient environment varies. Since the core mode is isolated within the fiber and not affected by any variations in the RI of the ambient environment, the phase difference between the core and cladding modes would be changing, which would cause a resultant shifting of the interference peaks or dips in the transmission spectrum.

With the deposition of a thin film of CNTs on the PCF as shown in Fig. 1(b), the sensing scheme is further modified. The deposition of the CNT thin film causes the shifting of the light energy of the cladding modes towards the radial edge of the PCF cladding, thus increasing the interaction of the cladding modes with the ambient environment [25]. CNTs are also known to be a material with high RI and high light absorbance [34], and the high RI would cause a change in the effective index of the cladding modes and also affect the intensity of the evanescent waves of the 
cladding modes at the PCF surface. This would result in a change in the wavelengths and extinction ratios of the interference peaks and dips of the transmitted spectrum after the deposition of the CNTs. When the CNT-deposited PCF is subjected to variations in RI in the ambient environment, the amount of reflectance of the intensity of the evanescent waves of the cladding modes can be given by [35]:

$$
R=\left|\frac{r_{23}+r_{34} e^{-i \tilde{k}_{C N T}}}{1+r_{23} r_{34} e^{-i \tilde{k}_{C N T}}}\right|^{2}
$$

where

$$
\begin{gathered}
r_{2,3}=\frac{n_{\text {clad }}-\tilde{n}_{C N T}}{n_{\text {clad }}+\tilde{n}_{C N T}} \\
r_{3,4}=\frac{\tilde{n}_{C N T}-n_{a m}}{\tilde{n}_{C N T}+n_{a m}} \\
\tilde{k}_{C N T}=\frac{4 \pi \tilde{n}_{C N T} d_{C N T}}{\lambda}=\frac{4 \pi n d_{C N T}}{\lambda}-i \frac{4 \pi k d_{C N T}}{\lambda}=\beta_{C N T}-i a d_{C N T}
\end{gathered}
$$

where $a=4 \pi k / \lambda$ is the absorption coefficient of the CNT layer, $\tilde{n}_{C N T}=n-i k, n_{\text {clad }}$ and $n_{a m}$ are the RIs of the cladding and the ambient environment, respectively. $r_{x, y}$ in equations (3) and (4) is the amplitude reflection coefficient of the interface between different dielectric layers $x$ and $y$. As shown in Fig. 1(b), the layers 1, 2, 3, and 4 correspond to that of the fiber core, fiber cladding, CNT layer and the ambient environment, respectively. From equations (2) to (5), it can be seen that the value of $R$ would vary with $n_{a m}$. Hence, as the RI of the ambient environment varies, so would the intensity of the evanescent wave of the cladding modes, which would result in a varying of the intensity of the cladding modes, causing a variation in the amplitudes of the interference peaks and dips. 


\section{Fabrication of Sensing Element}

The experimental setup for deposition of the CNT thin film is shown in Fig. 2(a). To fabricate the sensing element, a piece of PCF (NKT Photonics LMA-10) was stripped off its polymer jacket prior to splicing a $20 \mathrm{~mm}$ segment in between the input and output SMF fiber patch cords. Light from an ASE source (Optical Link Limited CL 15-16ASE) was coupled into the input SMF. The transmitted spectrum was monitored at the output SMF using an optical spectrum analyzer (OSA, Ando AQ 6317B) with a resolution of $0.1 \mathrm{~nm}$. The intensity of the spectrum was measured in the logarithmic scale instead of the linear scale as the intensity variations of the interference peaks and dips were very wide in the linear scale.

The CNT solution was prepared by first dispersing CNT powder into Dimethylformamide (DMF), before sonicating in a water bath. As shown in Fig. 2(a), a spray coating technique was then used to evenly deposit a thin film of CNTs around the fiber to fabricate the sensing element. The deposition mechanism relies solely on the cold transfer of CNTs onto the fiber surface [30]. This implies that no other specialized chambers are required to create an optimal environment for the deposition process, enhancing its cost effectiveness. It should be pointed out that the transmission spectrum was monitored throughout the deposition process. This was to control the amount of CNTs deposited to ensure that the final spectrum would retain suitable interference peaks and dips for sensing applications. The transmission spectrum of the CNT-deposited sensing element is depicted by the solid line in Fig. 3. As can be observed, there is a shift in the wavelengths of the interference peaks and dips as well as a variation in their intensity as compared to the bare sensing element which is depicted by the dotted line. This observation is expected based on equation (1) where the high RI of the CNTs would cause a change in the 
effective indices of the cladding modes, leading to a shift in wavelength of the resultant spectrum. The microscopy images of the sensing element before and after the deposition process can be seen in Figs 4(a) and 4(b). It can be seen that the CNTs were evenly deposited along the PCF, and have darkened the PCF fiber.

\section{Experiment and Results}

In order to test the behavior of the sensing element to the variations in RI of the ambient environment, saturated sucrose solution was first prepared by dissolving sugar into de-ionized water. Portions of the saturated solution were then extracted and diluted with de-ionized water to form different sucrose solutions of different sugar concentrations. The RI of each solution was measured using a digital refractometer (Kruss DR201-95) with a resolution of 0.0001. To avoid any temperature induced variations in the RI measurements, all measurements were carried out in a controlled environment at a constant temperature of $23.0 \pm 0.1{ }^{\circ} \mathrm{C}$. The experimental setup for RI measurement is shown in Fig. 2(b). The fabricated sensing element was placed over a glass slide and approximately $1 \mathrm{ml}$ of each sucrose solution was dropped on it. Care was taken to ensure that the entire sensing element was immersed in the sucrose solution and held straight throughout the entire measurement procedure. This would ensure that there would not be any bend induced perturbations on the sensing element which would affect the transmitted spectrum. At the input end, light from the ASE source was coupled through the sensing element and the transmitted spectrum was monitored by the OSA at the output end. The sensing element was thoroughly rinsed with de-ionized water and left to dry after each measurement was taken. It was ensured that the spectrum in air (solid line in Fig. 3) was recovered after each measurement before the subsequent measurement was carried out. 
Before deposition of CNTs, the spliced bare PCF was first exposed to different RIs. As shown in equation (1) and also previously in ref. [11], the behavior of the interference peaks or dips of a bare PCF in a MZI configuration would exhibit a red shift as the RI of the ambient environment increases. Fig. 5 plots the variation in wavelength of the interference dip at $1579 \mathrm{~nm}$. The maximum resolution for the RI range of 1.33 to 1.38 was calculated to be $2.0 \times 10^{-3}$ while the maximum resolution for the RI range of 1.38 to 1.42 was calculated to be $3.8 \times 10^{-4}$. These values are similar to that reported in ref. [11]. Fig. 6 shows spectral the behavior of the CNTdeposited PCF sensing element to variations in RI of the ambient environment. The interference dip at $1580.56 \mathrm{~nm}$ as shown in Fig. 3 was chosen as the reference dip due to its high extinction ratio. The sensor was found to display a clear and distinct change in intensity for each of the RI values used in the measurement. The attenuation of the interference dip decreases with increasing RI. This is in agreement with equations (2) to (5) where a variation in the RI of the ambient environment would result in a variation in $R$. The increase in RI of the ambient $\underline{\text { environment would cause a decrease in }} \underline{R}$ for RIs below that of the cladding. This indicates that less power from the evanescent waves are reflected back, resulting in a decrease in the amount of

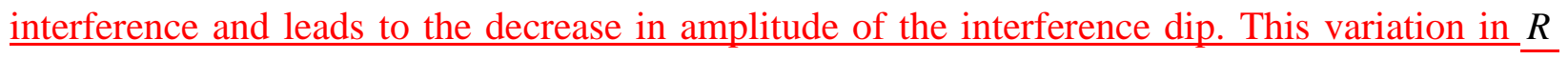
would be greater than that of the uncoated PCF where the intensity of the cladding modes at the interface of the fiber cladding and the ambient environment would depend only on Fresnel reflection. Hence, the CNT thin film would enhance the reflectance of the intensity of the cladding modes and cause a larger change in the intensity of the interference peaks and dips for different RIs of the ambient environment. The high RI of CNT would also result in a higher cladding effective index for the cladding modes as shown in equation (1), which leads to little variation in the cosine terms as the RI of the ambient environment varies. The transmitted 
spectrum would then exhibit little variation in the wavelength of the interference peaks and dips.

As shown in Fig. 6, the interference dip exhibited little deviation in wavelength throughout the entire measurement process. The slight deviation in wavelength observed can be attributed to the different volumes of each solution, resulting in a different amount of strain on the sensing element. It should also be pointed out that due to the nature of the sensing scheme, it was possible to take all measurements continuously and no reference measurements were needed before a particular measurement could be determined. This is an advantage over other reported sensors whose sensing schemes involve wavelength shifts of the interference spectrum with low FSR, to variations in the ambient environment $[3,5,7$, and 11$]$. Though highly sensitive, the detection range of these sensors is limited by the FSR and require reference values to be made in order to measure wider detection ranges. It can be seen that due to the sensor responding to different RIs with variations in intensity, a band pass filter and a power meter can be used instead of a costly OSA to detect the intensity variations. The sensing element can be cascaded with the band pass filter which would only allow a range of wavelengths (that of the chosen interference dip in this case) to transmit. The resultant transmitted light can then be detected by the power meter, hence providing a relatively cost effective sensing configuration. However, the insertion loss of the band pass filter has to be accounted for if such a sensing configuration were to be implemented as the output intensity still has to be within the detectable range of the power meter.

A second set of measurements, using the same experimental procedure as mentioned above, was carried out to test the repeatability of the sensor. Fig. 7 plots the change in the intensity of the interference dip with different RI values for both trials. The change in interference dip intensity was plotted relative to the data point with the lowest RI measurement for each respective trial. Based on the obtained data points (diamonds for trial 1 and hexagons for trail 2), a single fitting 
curve (solid line) was plotted. The maximum error for the repeatability of the trials was found to be $0.2 \mathrm{~dB}$ which can be attributed to the measurement errors by the OSA and slight power fluctuations in the ASE source. It can be seen that the intensity varies nonlinearly with the change in RI of the ambient environment. This nonlinear behavior of intensity with RI has been demonstrated in [4] and [29], and can also be explained by equation (2) where the amount of reflectance of the evanescent wave of the cladding modes varies nonlinearly with the RI of the ambient environment. These results show that the sensor exhibited consistent behavior to variations in RI and also demonstrated relatively good repeatability. The sensor registered a sensitivity of $19.4 \mathrm{~dB} /$ RIU and a sensitivity of $24.2 \mathrm{~dB} / \mathrm{RIU}$ within the RI ranges of 1.33 to 1.38 and 1.38 to 1.42 , respectively. The resolution of the sensor can be calculated to be approximately

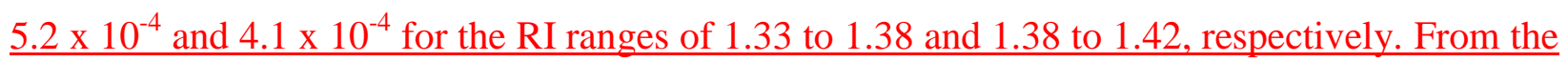
$\underline{\text { results, it can be observed that the resolution of the sensor is improved by an order of magnitude }}$ as compared to the bare PCF sensing element for the RI range of 1.33 to 1.38 . The sensor is also comparable in performance to other CNT based fiber sensors reported in [18], [20] and [29]. In $[18],[20]$ and $[29]$ the resolutions of the sensors were reported to be in the order of $10^{-4}$ and is matched with the CNT-deposited PCF.

In order to investigate the reproducibility of the fiber sensor, a second CNT-deposited PCF was fabricated using the same fabrication method. The deposited CNT thin film was inferred to be thinner than that of the first CNT-deposited PCF, exhibiting a less significant change in the intensity of the interference dip as similarly shown in Fig.3. The variation in intensity of the interference dip of the second CNT-deposited PCF (PCF 2) is also plotted in Fig. 7. A single fitting curve (dotted line) was also plotted through the data points (circles) as shown in the figure. 
It can be observed that the second CNT-deposited PCF exhibited a lower sensitivity as compared

to the first one. Hence, it can be estimated that a thicker CNT layer could improve the sensitivity.

The PCF has also been shown to be relatively insensitive to changes in the temperature of the surroundings, exhibiting a sensitivity of $0.019 \mathrm{~nm} /{ }^{\circ} \mathrm{C}$ [12]. This would make the sensor relatively immune to any unwanted thermal cross-sensitivity issues. As compared to the CNTdeposited LPFG described in ref. [29], this would make the CNT-deposited PCF more advantageous as a RI sensor. Due to the nature of the sensing scheme, it is also possible to simultaneously measure a second parameter, like strain, through variations in the wavelength. From equations (2) to (5), it can be seen that the CNT thin film is generally unaffected by the amount of strain on the CNT-deposited PCF. Hence the behavior of the sensor to variations in strain would generally be the same as that when using a bare PCF as given in reference [32]. This would allow the CNT-deposited PCF to measure two parameters simultaneously as the sensor would be expected to demonstrate a wavelength shift with variations in strain, and an intensity difference to variations in RI, thus allowing for a dual parameter sensor.

\section{Conclusion}

In summary, we have demonstrated a novel and relatively effective carbon nanotube (CNT) deposited photonic crystal fiber (PCF) sensor featuring a Mach-Zehnder interferometer (MZI) configuration. Such a scheme requires no modification of the fiber structure and thus preserves its mechanical strength. The sensor was able to display clear and distinct intensity changes with little wavelength variation for different solutions with different refractive index (RI) values. This would allow the sensor to gain immunity from free spectral range (FSR) limitations. The sensor exhibited a sensitivity of $19.4 \mathrm{~dB} /$ RIU within the RI range of 1.33 to 1.38 and a sensitivity of $24.2 \mathrm{~dB} / \mathrm{RIU}$ within the RI range of 1.38 to 1.42 . There was good repeatability in terms of the 
fabrication of the sensor which can be seen from the second CNT-deposited PCF which exhibited the same trend in the variation of the intensity of the interference dip with RI.

\section{Acknowledgment}

This work was partially supported by Academic Research Fund Tier 1 Grant (RG22/10) of Ministry of Education (MOE) and Nanyang Technological University, Singapore. 


\section{References}

[1] P. Lu, L. Q. Men, K. Sooley, and Q. Y. Chen, "Tapered fiber Mach-Zehnder interferometer for simultaneous measurement of refractive index and temperature," Appl. Phys. Lett., vol. 94, no. 13, Mar. 2009.

[2] P. F. Wang, G. Brambilla, M. Ding, Y. Semenova, Q. Wu, and G. Farrell, "High-sensitivity, evanescent field refractometric sensor based on a tapered, multimode fiber interference," Opt. Lett., vol. 36, no. 12, pp. 2233-2235, Jun. 2011.

[3] W. B. Ji, H. H. Liu, S. C. Tjin, K. K. Chow, and A. Lim, "Ultrahigh sensitivity refractive index sensor based on optical microfiber," IEEE, Photon. Technol. Lett., vol. 24, no. 20, pp. 1872-1874, Oct. 2012.

[4] S. Silva, O. Frazão, J. L. Santos, and F. X. Malcata, "A reflective optical fiber refractometer based on multimode interference," Sens. Actuators, B, vol. 161, no. 1, pp. 88-92, Jan. 2012.

[5] J. Wang, Y. Jin, Y. Zhao, and X. Dong, "Refractive index sensor based on all-fiber multimode interference," Optik, vol. 124, no. 14, pp. 1845-1848, Jul. 2013.

[6] C. Gouveia, G. Chesini, C. M. B. Cordeiro, J. M. Baptista, and P. A. S. Jorge, "Simultaneous measurement of refractive index and temperature using multimode interference inside a high birefringence fiber loop mirror," Sens. Actuators, B, vol. 177, pp. 717-723, Feb. 2013.

[7] G. Yin, S. Lou, and H. Zou, "Refractive index sensor with asymmetrical fiber Mach-Zehnder interferometer based on concatenating single-mode abrupt taper and core-offset section," Opt. Laser Technol., vol. 45, pp. 294-300, Feb. 2013. 
[8] W. C. Wong, C. C. Chan, Y. F. Zhang, and K. C. Leong, "Miniature single-mode fiber refractive index interferometer sensor based on high order cladding mode and core-offset," IEEE Photon. Technol. Lett., vol. 24, no. 5, pp. 359-361, Mar. 2012.

[9] C. C. C. Lam, R. Mandamparambil, T. Sun, K. T. V. Grattan, S. V. Nanukuttan, S. E. Taylor, P. A. Muhammed Basheer, "Optical fiber refractive index sensor for chloride ion monitoring," IEEE Sensors J., vol. 9, no. 5, pp. 525-532, May. 2009.

[10] H. Meng, W. Shen, G. Zhang, C. Tan, and X. Huang, "Fiber Bragg grating-based fiber sensor for simultaneous measurement of refractive index and temperature," Sens. Actuators, B, vol. 150, no. 1, pp. 226-229, Sep. 2010.

[11] R. Jha, J. Villatoro, G. Badenes, and V. Pruneri, "Refractometry based on a photonic crystal fiber interferometer," Opt. Lett., vol. 34, no. 5, pp. 617-619, Mar. 2009.

[12] W. C. Wong, C. C. Chan, L. H. Chen, Z. Q. Tou, and K. C. Leong, "Highly sensitive miniature photonic crystal fiber refractive index sensor based on mode field excitation," Opt. Lett., vol. 36, pp. 1731-1733, May 2011.

[13] K. M. Tan, C. M. Tay, S. C. Tjin, C. C. Chan, and H. Rahardjo, "High relative humidity measurements using gelatin coated long-period grating sensors," Sens. Actuators, B, vol. 110, no. 2, pp. 335-341, Oct. 2005.

[14] M. Smietana, J. Szmidt, M. L. Korwin-Pawlowski, W. J. Bock, and J. Grabarczyk, "Application of diamond-like carbon films in optical fibre sensors based on long-period gratings," Diamond Relat. Mater., vol. 16, no. 4-7, pp. 1374-1377, Apr. 2007 
[15] Q. Li, X. L. Zhang, Y. S. Yu, Y. Qian, W. F. Dong, Y. Li, J. G. Shi, J. T. Yan, H. Y. Wang, "Enhanced sucrose sensing sensitivity of long period fiber grating by self-assembled polyelectrolyte multilayers," React. Funct. Polym., vol. 71, no. 3, pp. 335-339, Mar. 2011.

[16] S. Korposh, R. Selyanchyn, W. Yasukochi, S. W. Lee, S. W. James, and R. P. Tatam, "Optical fibre long period grating with a nanoporous coating formed from silica nanoparticles for ammonia sensing in water," Mater. Chem. Phys., vol. 133, no. 2-3, pp. 784-792, Apr. 2012.

[17] A. Cusano, M. Consales, A. Cutolo, M. Penza, P. Aversa, M. Giordano, A. Guemes, "Optical probes based on optical fibers and single-walled carbon nanotubes for hydrogen detection at cryogenic temperatures," Appl. Phys. Lett., vol. 89, no. 20, pp. 201106-201106-3, Nov. 2006.

[18] M. Consales, A. Crescitelli, S. Campopiano, A. Cutolo, M. Penza, P. Aversa, M. Giordano, A. Cusano, "Chemical detection in water by single-walled carbon nanotubes-based optical fiber sensors," IEEE Sensors J., vol. 7, no. 7-8, pp. 1004-1005, Jul 2007.

\section{[19] M. Consales, A. Cutolo, M. Penza, P. Aversa, G. Cassano, M. Giordano, et al., "Carbon} nanotubes coated acoustic and optical VOCs sensors: towards the tailoring of the sensing performances," IEEE Trans. Nanotechnol., vol. 6, no. 6, pp. 601-612, Nov. 2007.

[20] M. Consales, A. Crescitelli, M. Penza, P. Aversa, P. D. Veneri, M. Giordano, A. Cusano, "SWCNT nano-composite optical sensors for VOC and gas trace detection," Sens. Actuators, B, vol. 138, no. 1, pp. 351-361, Apr. 2009 
[21] J. R. Zhao, X. G. Huang, W. X. He, and J. H. Chen, "High-resolution and temperatureinsensitive fiber optic refractive index sensor based on fresnel reflection modulated by FabryPerot interference," J. Lightw. Technol., vol. 28, no. 19, pp. 2799-2803, Oct. 2010.

[22] K. S. Kim, Y. Mizuno, M. Nakano, S. Onoda, and K. Nakamura, "Refractive index sensor for liquids and solids using dielectric multilayer films deposited on optical fiber end surface," IEEE Photon. Technol. Lett., vol. 23, no. 20, pp. 1472-1474, Oct. 2011.

[23] L. H. Chen, T. Li, C. C. Chan, R. Menon, P. Balamurali, M. Shaillender, B. Neu, X. M. Ang, P. Zu, W. C. Wong, K. C. Leong "Chitosan based fiber-optic Fabry-Perot humidity sensor," Sens. Actuators, B, vol. 169, pp. 167-172, Jul. 2012.

[24] H. Tu, T. Sun, and K. T. Grattan, "SPR-based optical fiber sensors using gold-silver alloy particles as the active sensing material," IEEE Sensors J., vol. 13, no. 6, pp. 2192-2199, Jun. 2013.

[25] M. Smietana, D. Brabant, W. J. Bock, P. Mikulic, and T. Eftimov, "Refractive-index sensing with inline core-cladding intermodal interferometer based on silicon nitride nano-coated photonic crystal fiber," J. Lightw. Technol., vol. 30, no. 8, pp. 1185-1189, Apr. 2012.

[26] Z. Q. Tou, C. C. Chan, W. C. Wong, and L. H. Chen, "Fiber optic refractometer based on cladding excitation of localized surface plasmon resonance," IEEE Photon. Technol. Lett., vol. 25, no.6, pp. 556-559, Mar. 2013.

[27] T. Li, X. Y. Dong, C. C. Chan, K. Ni, S. Q. Zhang, and P. P. Shum, "Humidity sensor with a PVA-coated photonic crystal fiber interferometer," IEEE Sensors J., vol. 13, no.6, pp. 2214-2216, Jun. 2013. 
[28] W. C. Wong, C. C. Chan, L. H. Chen, T. Li, K. X. Lee, and K. C. Leong, "Polyvinyl alcohol coated photonic crystal optical fiber sensor for humidity measurement," Sens. Actuators, B, vol. 174, pp. 563-569, Nov 2012.

[29] Y. C. Tan, W. B. Ji, V. Mamidala, K. K. Chow, and S. C. Tjin, "Carbon-nanotubedeposited long period fiber grating for continuous refractive index sensor applications," Sens. Actuators, B, vol. 196, pp. 260-264. Jun. 2014.

[30] K. K. Chow, S. Yamashita, and S. Y. Set, "Four-wave-mixing-based wavelength conversion using a single-walled carbon-nanotube-deposited planar lightwave circuit waveguide," Opt. Lett., vol. 35, no. 12, pp. 2070-2072. Jun. 2010.

[30] H. Y. Choi, M. J. Kim, and B. H. Lee, "All-fiber Mach-Zehnder type interferometers formed in photonic crystal fiber," Opt. Express, vol. 15, no. 9, pp. 5711-5720, Apr. 2007.

[32] J. Villatoro, V. Finazzi, V. P. Minkovich, V. Pruneri, and G. Badenes, "Temperatureinsensitive photonic crystal fiber interferometer for absolute strain sensing," Appl. Phys. Lett., vol. 91, no. 9, pp. 091109-091109-3, Aug. 2007.

[33] L. M. Hu, C. C. Chan, X. Y. Dong, Y. P. Wang, P. Zu, W. C. Wong, et al., "Photonic crystal fiber strain sensor based on modified Mach-Zehnder interferometer," IEEE Photon. J., vol. 4, no. 1, pp. 114-118, Feb. 2012.

[34] V. A. Margulis and E. A. Gaiduk, "Nature of near-infrared absorption in single-wall carbon nanotubes," Phys. Lett. A, vol. 281, no. 1, pp. 52-58, Mar. 2001.

[35] H. A. Macleod, Thin-film optical filters/ H. Anglus Macleod, 4th ed. Boca Raton, FL: CRC Press/Taylor \& Francis, c2010., 2010. 


\section{Figure captions:}

Figure 1 Schematic illustration of the (a) bare and (b) carbon nanotube-deposited (CNTdeposited) photonic crystal fiber (PCF) sensor featuring a Mach-Zehnder Interferometer (MZI).

Figure 2 Experimental setup of (a) CNT deposition onto the PCF and (b) RI sensing of the test solution.

Figure 3 Transmission spectrum of the PCF before CNT deposition (dotted line) and after CNT deposition (solid line).

Figure 4 Optical microscopic images of the PCF (a) before CNT deposition and (b) after CNT deposition under the same magnification.

Figure 5 Plot of Intensity change of the interference dip against RI of the test solution for the PCF before CNT deposition.

Figure 6 Transmission spectra showing the interference dip of the CNT-deposited PCF with test solutions of different RIs.

Figure 7 Plot of the intensity change of the interference dip against RI of the test solution. A fitting curve (solid line) plotted through the data points for the first trial (diamonds) and second trial (hexagons) of CNT-deposited PCF 1 to show the behavior of the sensing element to variations in RI. The data points (circles) of CNT-deposited PCF 2 are also shown with a fitting curve (dotted line). $\mathrm{I}_{1}$ and $\mathrm{I}_{2}$ are the equations of the fitting curves for the CNT-deposited PCF 1 and CNTdeposited PCF 2, respectively. 


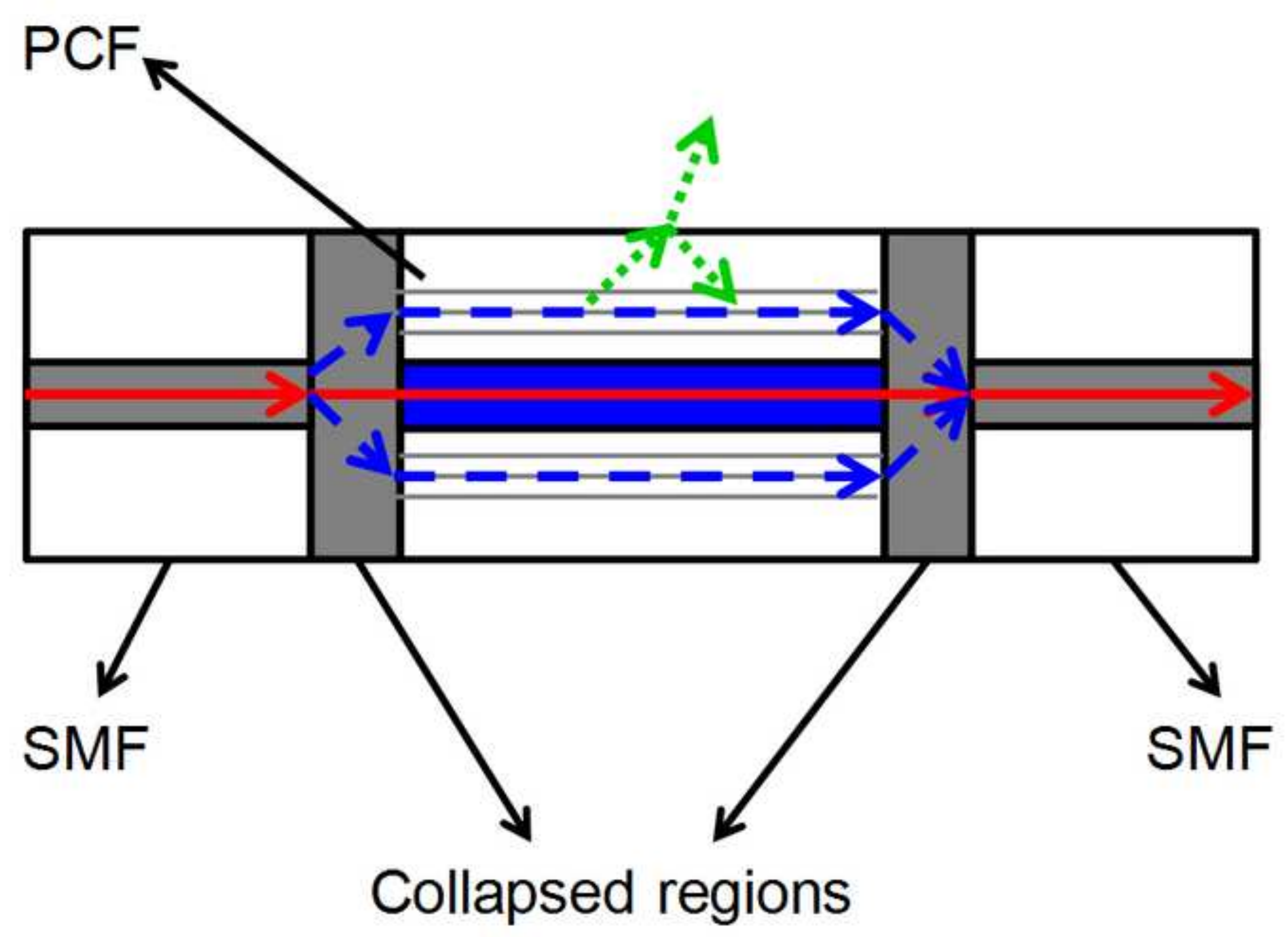




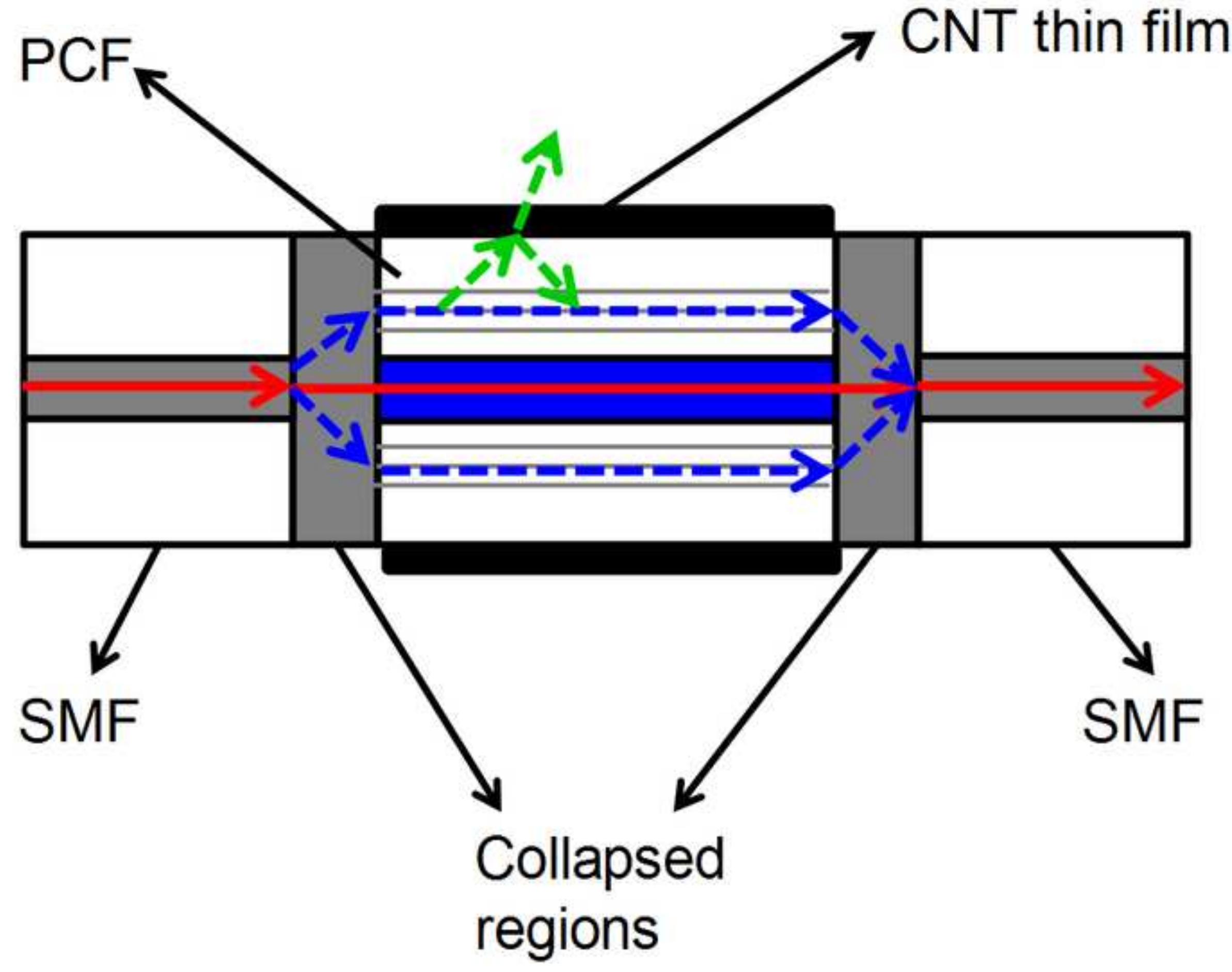




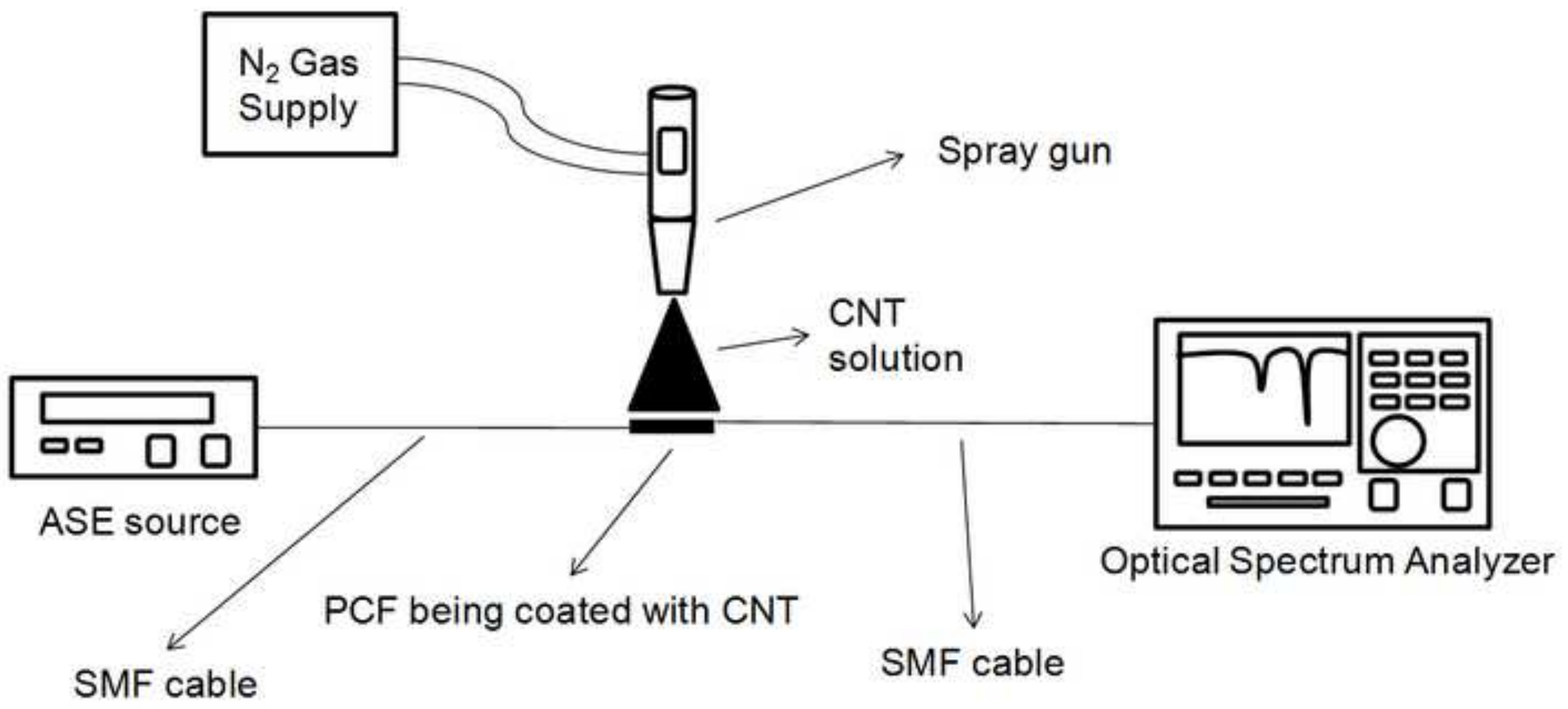




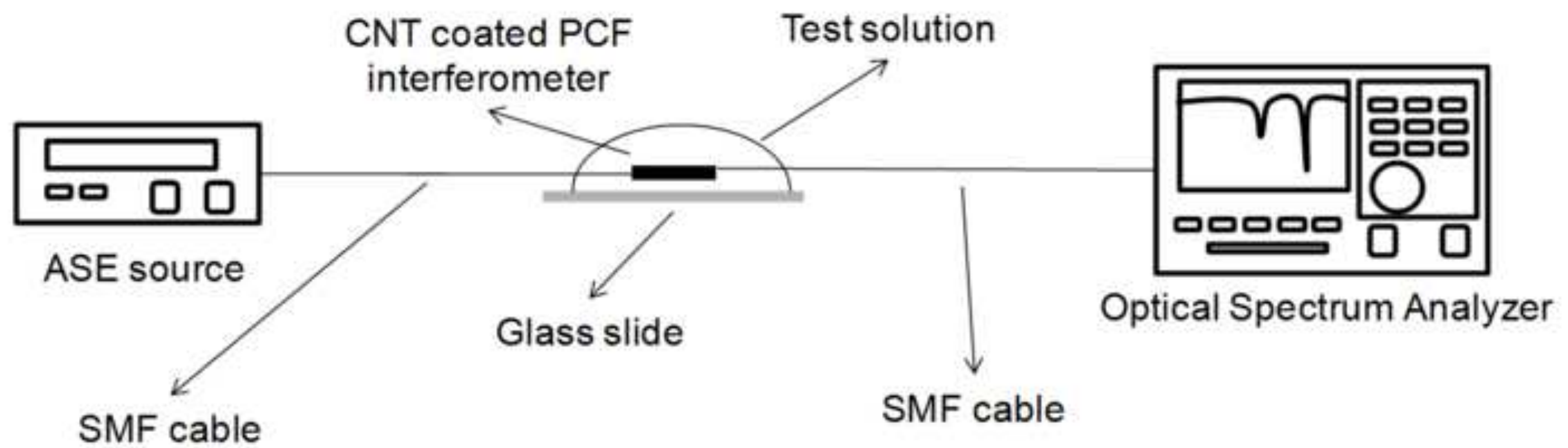


Figure(s)
Click here to download high resolution image

(a)

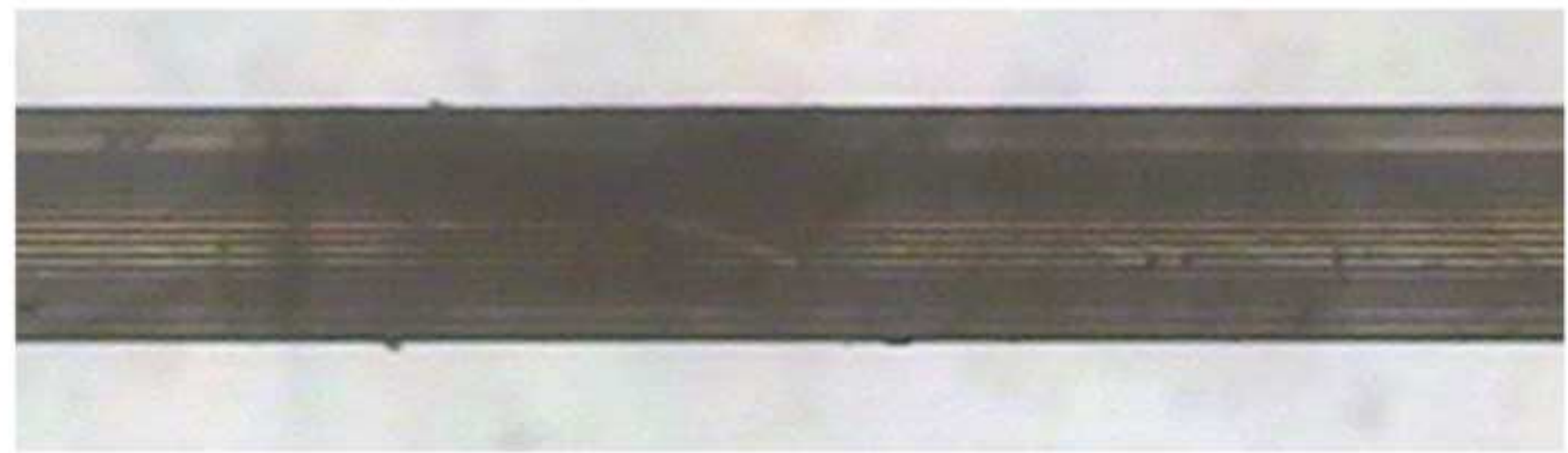

(b)

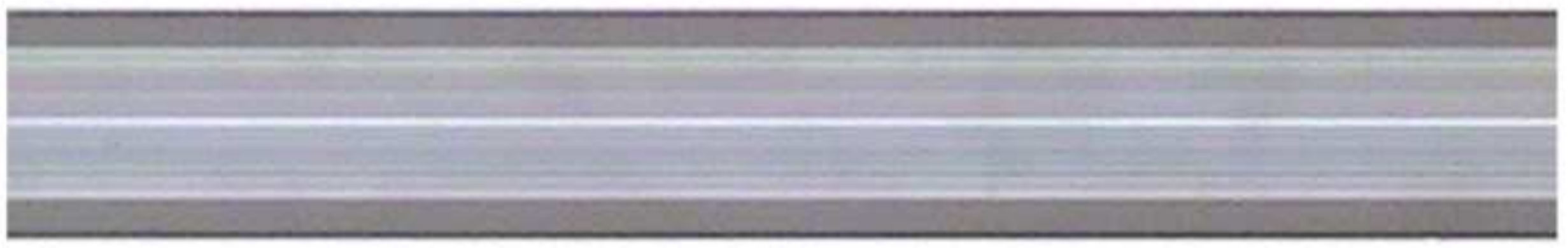




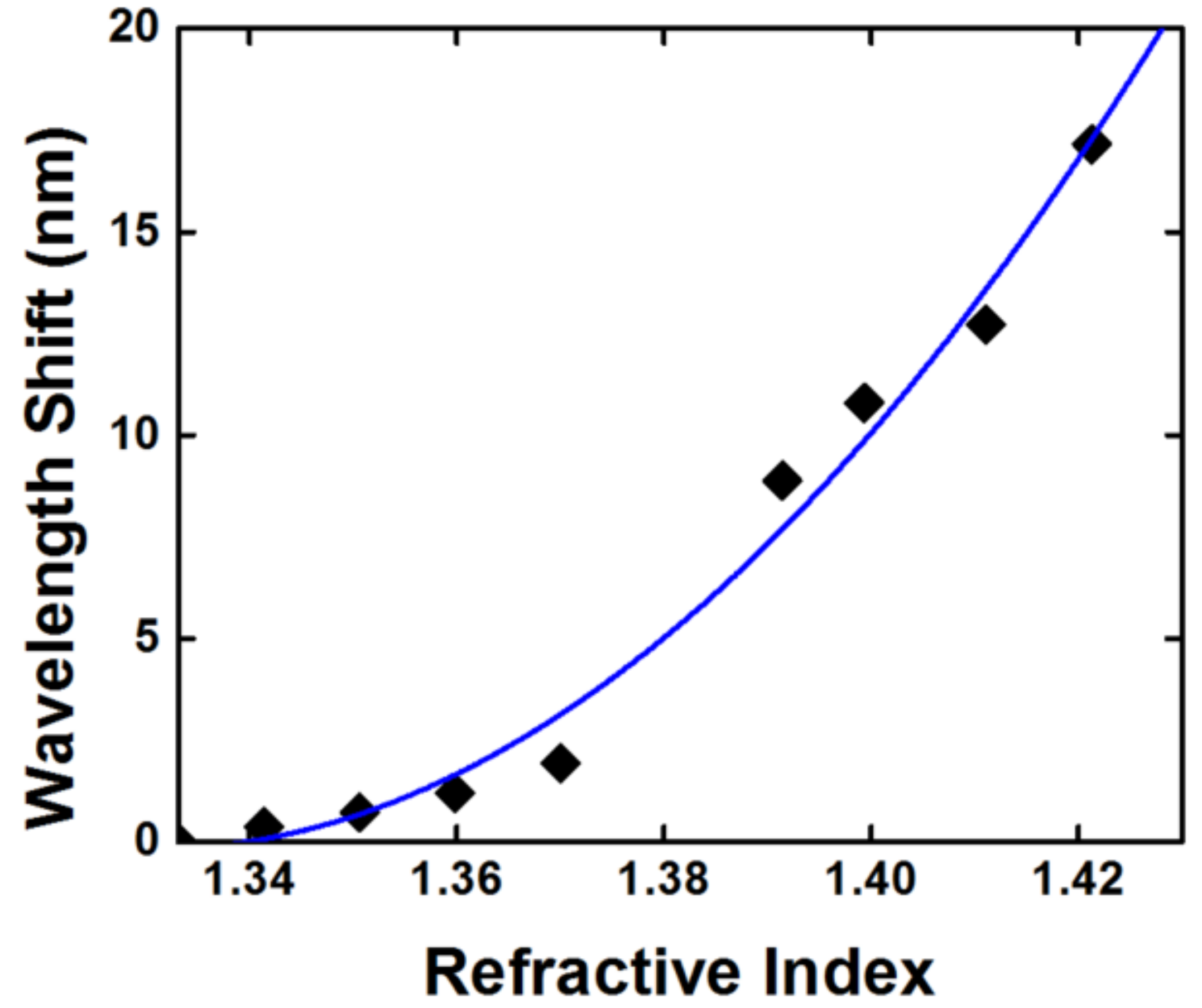

Figure(s)
Click here to download high resolution image 


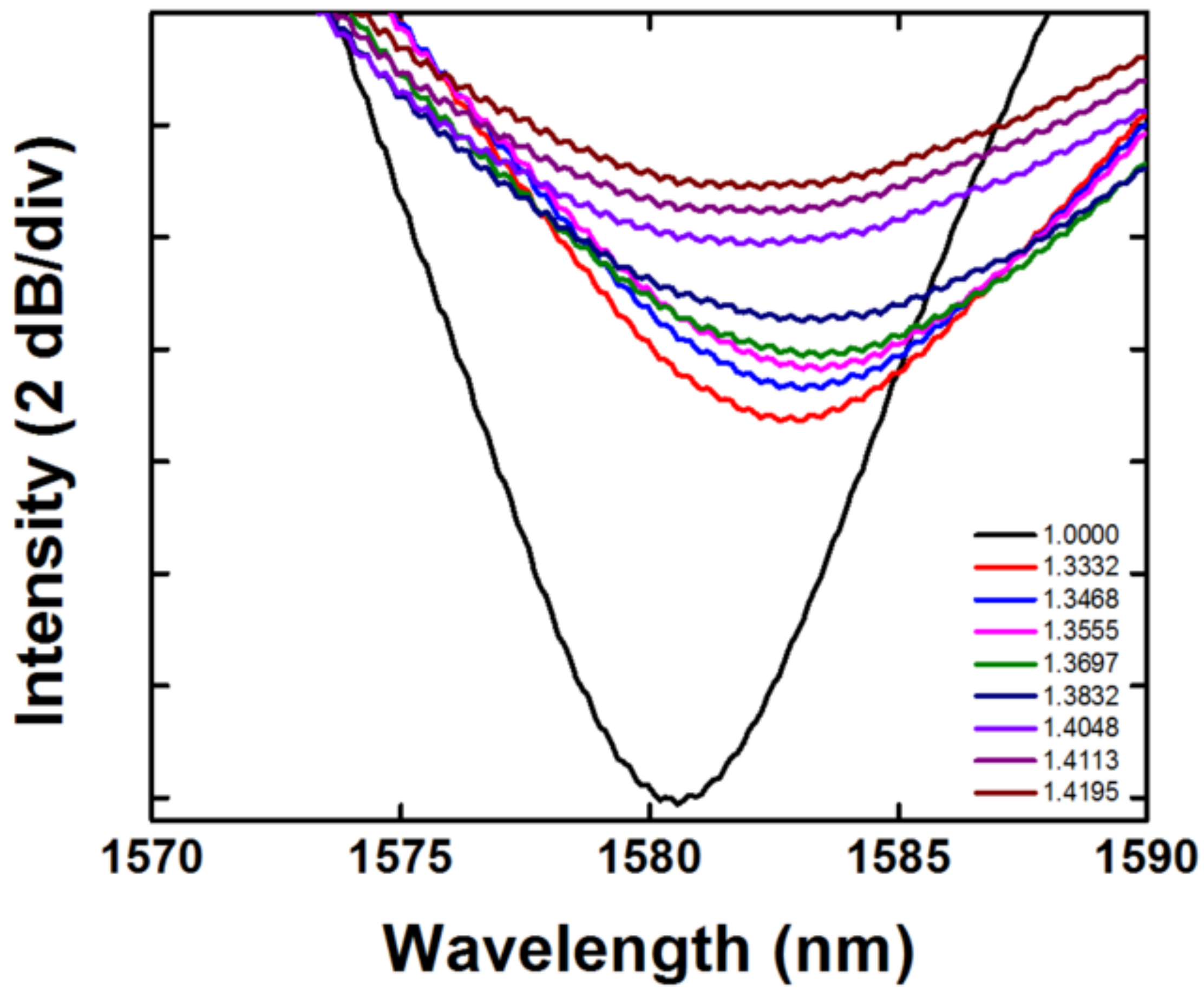




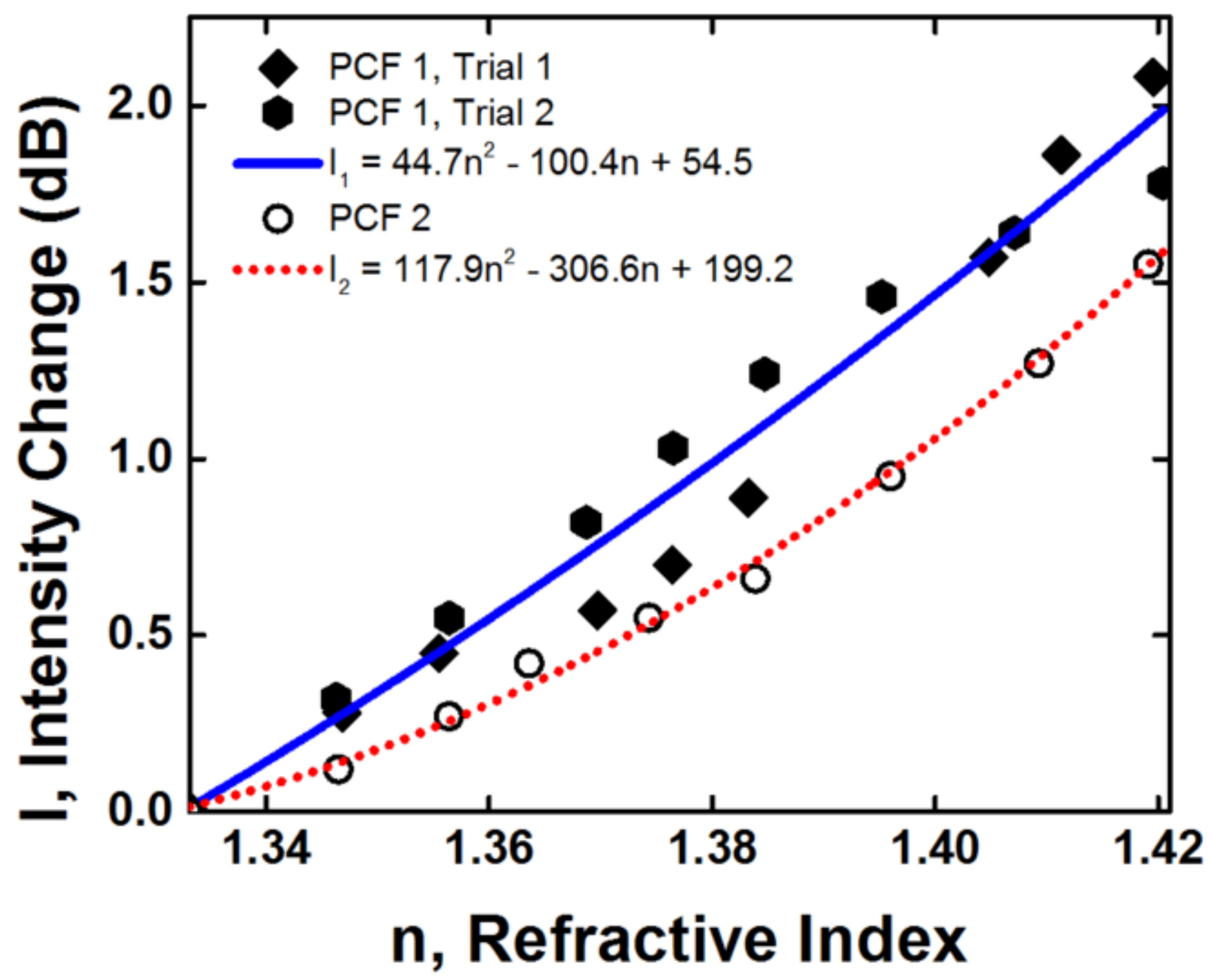

